\title{
Pertrochantäre Femurfraktur
}

\author{
Thomas Mittlmeier
}

\section{Einleitung}

Die Inzidenz instabiler pertrochantärer Femurfrakturen steigt mit Zunahme der Lebenserwartung unserer älteren Patienten bei konkomitanter Osteoporose stetig an. Während sich in Deutschland und Europa intramedulläre Rekonstruktionsnägel bei der Versorgung der $\mathrm{AO} /$ OTA-31-A2- und A3-Frakturen großer und zunehmender Beliebtheit erfreuen, lässt die Studienlage der evidenzbasierten Medizin im Vergleich zum gängigen Alternativverfahren, nämlich der Applikation der dynamischen Hüftschraube, bislang keine sicheren Vorteile intramedullärer Nagelsysteme erkennen [1, 2,4-6]. Wenngleich das biomechanische Konzept intramedullärer Systeme günstig für eine umgehende postoperative Mobilisation des Patienten unter Vollbelastung erscheint [3], ist die höhere Komplikationsrate der proximalen Femurnägel (Tab.1) - im Vergleich mit der DHS - bei der bisherigen Studienlage ein relevantes Argument zugunsten einer Favorisierung der DHS bei der Versorgung der häufigsten Frakturformen einer pertrochantären Femurfraktur [4]. Insbesondere das Risiko einer intra- oder postoperativen Femur-

\begin{tabular}{|c|c|}
\hline Verriegelungsbolzenbruch & $0,5-4,6 \%$ \\
\hline intraop. Schaftsprengung & $0,5-5 \%$ \\
\hline $\begin{array}{l}\text { Cut-out der Schenkelhals- } \\
\text { schraube }\end{array}$ & $3,2-4,2 \%$ \\
\hline Implantatversagen & $0-5 \%$ \\
\hline Nagelrandfraktur & $0-6 \%$ \\
\hline Malalignment/Malrotation & $1-15 \%$ \\
\hline
\end{tabular}

OP-JOURNAL 2008; 24: 222-223

(c) Georg Thieme Verlag KG Stuttgart • New York DOI 10.1055/s-2008-1039070
Tab. 2 Inzidenz implantatbezogener Femurfrakturen.

\begin{tabular}{ll}
\hline Implantat & $\begin{array}{l}\text { per } \mathbf{1 0 0 0} \\
\text { Personen/ } \\
\text { Jahr }\end{array}$ \\
\hline DHS & 4,46 \\
\hline kanülierte Schrauben & 4,50 \\
\hline TEP zementiert & 6,2 \\
\hline nicht zementierte TEP & 11,7 \\
\hline Gammanagel & $\mathbf{1 8 , 7}$ \\
\hline Revisionsarthroplastik & $\mathbf{2 2 , 4}$
\end{tabular}

Nach Robinson CM, Adams Cl, Craig M, Doward W, Clarke MCC, Auld J: J Bone Joint Surg [Am] 2002; 84: 1116-1122

fraktur - besonders auffällig bei der ursprünglichen Version des Gammanagels (Tab. 2) - wird in vergleichenden Studien konstatiert $[2,4]$. Inwieweit hier neben Implantat-, also designtypischen Konstruktionsmerkmalen auch OPtechnische Schwierigkeiten relevant sind, kann noch nicht definitiv beantwortet werden [5]. Fehlheilungen und periimplantäre Frakturen ziehen bei der instabilen Situation am proximalen Femur zwangsläufig Folgeeingriffe nach sich.

\section{Kasuistik}

Eine 66-jährige Frau mit Adipositas permagna (BMI 46,2), Diabetes mellitus II, essenzieller Hypertonie sowie chroni- scher zerebrovaskulärer Insuffizienz mit Schwindelneigung stürzte in der Häuslichkeit auf ihre rechte Hüfte und zog sich eine dislozierte pertrochantäre Femurfraktur (AO 31-A2.3) zu (Abb.1 a). Die Verletzung wurde gleichentags operativ versorgt: Auf dem Extensionstisch wurde eine geschlossene Reposition vorgenommen und ein biaxialer proximaler Rekonstruktionsnagel von Standardlänge (Targon PF [Aesculap, Tuttlingen] $130^{\circ}, 220 \mathrm{~mm}$, Durchmesser $10 \mathrm{~mm}$, einfache statische Verriegelung) implantiert (Abb. 1 b und c). Postoperativ konnte die Patientin bei primärer Wundheilung am Rollator auf Stationsebene mobilisiert werden. Eine AHB wurde von der Patientin abgelehnt. Die Entlassung erfolgte am 12. postoperativen Tag nach Hause. Die Patientin erreichte eigenen Angaben nach ihren Mobilisationsgrad wie vor dem Unfall. 15 Monate nach OP wollte die Patientin von einer Couch aufstehen und berichtete über heftige Schmerzen über der rechten Hüfte und der Trochanterregion, ein neuerliches Trauma war nicht erinnerlich. Die Röntgenaufnahme zeigte eine Ermüdungsfraktur des Implantats auf typischer Höhe (Eintritt Schenkelhalsschraube) bei offenkundiger Pseudarthrose im vormaligen Frakturbereich (Abb.2a). Binnen 48 Stunden erfolgte die Reoperation auf dem Extensionstisch mit Implantatentfernung, Aufbohren der intertrochantären Pseudarthrosenregion bis zu $15 \mathrm{~mm}$, Reposition und Reosteosynthese mittels einer Langnagelversion des iden-

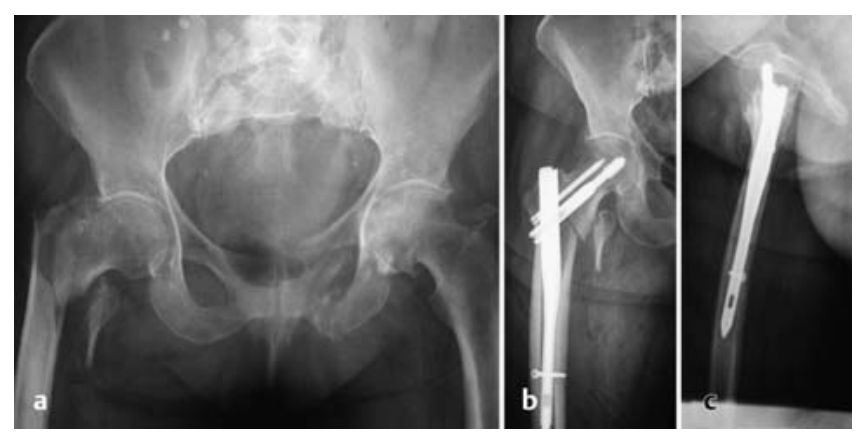

Abb. 1 a bis c Unfallbild (a) und postoperatives Versorgungsresultat (b,c). 


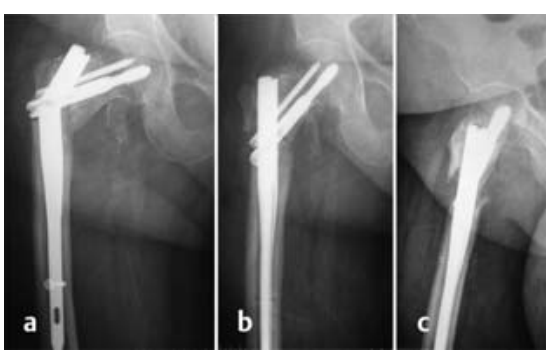

Abb.2a bis c a Implantatermüdung bei Pseudarthrose. b,c Revision mittels Langnagelversion.

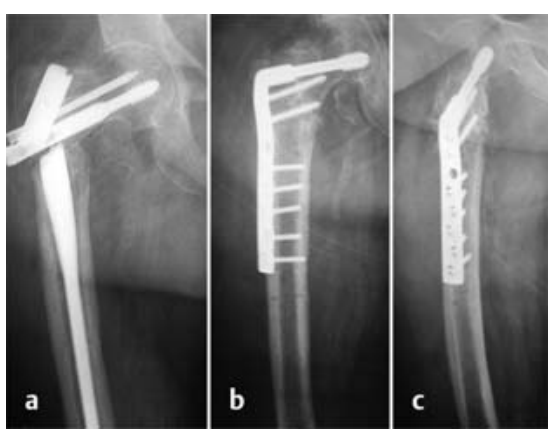

Abb. $\mathbf{3 a}$ bis c a Persistierende Pseudarthrose und erneutes Implantatversagen. b, c Revision mit extramedullärem Implantat (DCS).

tischen Implantattyps (Targon PF lang [Aesculap, Tuttlingen] $10 \times 380 \mathrm{~mm}$, distal statisch doppelt verriegelt) und autologer Spongiosaplastik vom rechten vorderen Beckenkamm (Abb. $2 \mathbf{b}$ und $\mathbf{c}$ ). Wiederum gelang bei ungestörter Wundheilung die Mobilisation am Rollator und die Entlassung am 17. postoperativen Tag nach Hause. Eine AHB wurde von der Patientin erneut abgelehnt.

Nur 6 Monate später wurde die Patientin nach einem weiteren Sturz wieder stationär aufgenommen. Radiomorphologisch manifestierte sich eine persistierende Pseudarthrose bei neuerlichem Implantatversagen (Abb.3a). Nach Implantatentfernung erfolgt nun ein Verfahrenswechsel auf eine extramedulläre Stabilisation mittels DCS und abermaliger autologer Spongiosaplastik kombiniert mit synthetischem Knochenersatzmaterial (Nanobone, Abb. $\mathbf{3 b}$ und c). Binnen 14 Tage erfolgte bei primärer Wundheilung unter Teilbelastung der rechten unteren Extremität mit $20 \mathrm{~kg}$ am Gehwagen die Verlegung in eine stationäre geriatrische Rehabilitationseinrichtung. Nur 3 Tage später wurde die Patientin nach einem Sturz im Badezimmer zurückverlegt. Die Patienten hatte sich eine periimplantäre Femurfraktur zugezogen (Abb.4a und b), die noch am Aufnahmetag mit einer 9-Loch-LISS versorgt wurde (Abb. 4c bis e). Postope-

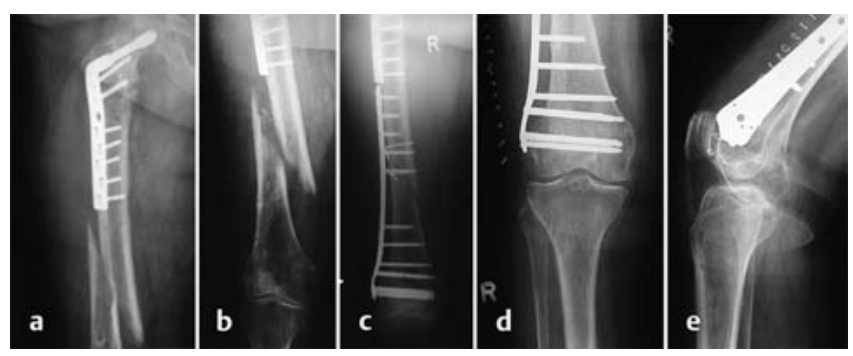

Abb. 4a bis e Periimplantäre Femurschaftfraktur $(\mathbf{a}, \mathbf{b})$ und Osteosynthese mittels LISS (c-e).

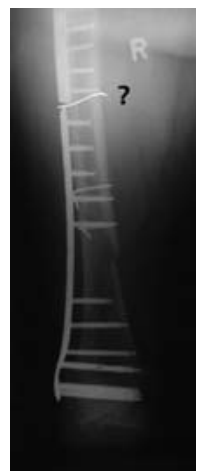

Abb. 5 Hohes Risikopotenzial einer interimplantären Femurfraktur.

rativ wurde die Patienten wiederum am Rollator unter $20 \mathrm{~kg}$ Teilbelastung mobilisiert. Das offenkundige Risiko einer potenziellen Refraktur durch Stresskonzentration am genuinen Knochen zwischen den beiden extramedullären Implantaten ist auf der a.-p. Aufnahme ablesbar (Abb. 5).

Eine Wiedervorstellung der Patientin erfolgte erst binnen Jahresfrist wegen einer nicht dislozierten Humeruskopffraktur, die funktionell-konservativ behandelt wurde. Die Patientin war laut Fremdangaben im Weiteren leidlich mit Rollatorunterstützung im häuslichen Umfeld mobil und verstarb 1,5 Jahre nach dem letzten Eingriff am rechten Femur wegen Herz-Kreislauf-Problemen.

\section{Fazit}

Ein manifester OP-technischer Fehler oder eine ungeeignete Implantatwahl ist am aufgezeigten Beispiel schwerlich ableitbar; allenfalls wäre diskutabel, ob ein Verfahrenswechsel auf ein extramedulläres System bereits nach der Erstmanifestation der Pseudarthrose sinnvoll gewesen wäre. Das Hauptproblem liegt hier zweifelsohne auf der Patientenseite, da die bekannte Sturzneigung (CVI) bei Adipositas permagna und ungünstige Heilungsvoraussetzungen (Diabetes mellitus, manifeste Osteoporose, Verweigerung einer AHB) den „Takt vorgaben" und die Folgeeingriffe diktierten. Grundsätzlich ist bei der Versorgungsstrategie derartiger Patienten abzuwägen, welches Verfahren bei neuerlichen Stürzen am besten geeignet ist, Stresskonzentrationen zu vermeiden. Im vorliegenden Falle hätte eine interimplantäre Fraktur in jedem Fall eine Entfernung beider einliegender extramedullärer Systeme bedeutet.

\section{Literatur}

1 Giraud B, Dehoux E, Jovenin N, Madi K, Harisboure A, Usandizaga G, Segal P. Comparaison vis-plaque dynamique et osteosynthese intramedullaire antegrade dans les fractures pertrochanteriennes: une etude prospective randomisee. Rev Chir Orthop Reparatrice Appar Mot 2005; 91: 732-736

2 Hesse B, Gächter A. Complications following the treatment of trochanteric fractures with the gamma nail. Arch Orthop Trauma Surg 2004; 124: 692-698

3 Pajarinen J, Lindahl J, Michelsson O, Savolainen V, Hirvensalo E. Pertrochanteric femoral fractures treated with a dynamic hip screw or a proximal femoral nail. A randomised study comparing post-operative rehabilitation. J Bone Joint Surg [Br] 2005; 87: 76-81

4 Parker MJ, Handoll HH. Gamma and other cephalocondylic intramedullary nails versus extramedullary implants for extracapsular fractures in adults. Cochrane Database Syst Rev 2008; 3: CD000093

5 Saudan M, Lubbecke A, Sadowski C, Riand N Stern R, Hoffmeyer P. Pertrochanteric fractures: is there an advantage to an intramedullary nail? A randomized, prospective study of 206 patients comparing the dynamic hip screw and proximal femoral nail. J Orthop Trauma 2002; 16: 386-393

6 Stern R. Are there advances in the treatment of extracapsular hip fractures in the elderly? Injury 2007; 38 (Suppl. 3): S77-S87

\section{Univ.-Prof. Dr. med. Thomas Mittlmeier Klinikdirektor}

Abteilung für Unfall- und Wiederherstellungschirurgie Chirurgische Klinik und Poliklinik der Universität Rostock Schillingallee 35 18055 Rostock

E-Mail: thomas.mittlmeier@ med.uni-rostock.de 